\title{
Effective Quantum Field Theory for the Thermodynamical Bethe Ansatz
}

\author{
Ivan Kostov \\ Institut de Physique Théorique, DSM, CEA, URA2306 CNRS, \\ Saclay, F-91191 Gif-sur-Yvette, France \\ E-mail: ivan.kostov@ipht.fr
}

ABstract: We construct an effective Quantum Field Theory for the wrapping effects in $1+1$ dimensional models of factorised scattering. The recently developed graph-theoretical approach to TBA gives the perturbative desctiption of this QFT. For the sake of simplicity we limit ourselves to scattering matrices for a single neutral particle and no bound state poles, such as the sinh-Gordon one. On the other hand, in view of applications to AdS/CFT, we do not assume that the scattering matrix is of difference type. The effective QFT involves both bosonic and fermionic fields and possesses a symmetry which makes it one-loop exact. The corresponding path integral localises to a critical point determined by the TBA equation.

Keywords: Bethe Ansatz, Integrable Field Theories, Topological Field Theories

ARXIV EPRINT: 1911.07343 


\section{Contents}

1 Introduction 1

2 Degrees of freedom of the effective QFT 3

2.1 Space and time wrapping operators 3

2.2 Operator form of the Bethe-Yang equations 6

$\begin{array}{lll}2.3 & \text { Free-field realisation } & 7\end{array}$

3 Partition function on the torus $\quad 8$

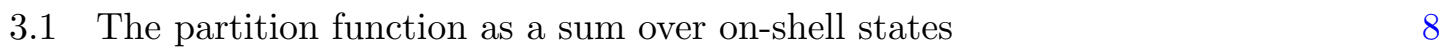

$\begin{array}{llr}3.2 & \text { Rewriting the sum as a contour integral } & 9\end{array}$

$\begin{array}{ll}3.3 \text { Continuous spectrum approximation } & 11\end{array}$

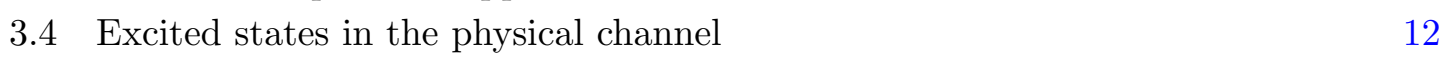

4 Path integral and localisation $\quad 13$

5 Example: the Sinh-Gordon model $\quad 15$

$\begin{array}{llr}6 & \text { Concluding remarks } & 18\end{array}$

$\begin{array}{ll}\text { A Conventions about the scattering in physical and mirror kinematics } & 20\end{array}$

B Operator representation of the Gaudin determinant 20

\section{Introduction}

In $1+1$ dimensional field-theoretical models with factorised scattering, the momenta and the energies of the particles forming a multi-particle excitation are not changed by the Hamiltonian evolution. This property makes possible to use the notion of a particle with given rapidity even after the theory is confined on a circle with asymptotically large circumference $R$. Asymptotically large signifies much larger than the correlation length, so that the exponential effects can be neglected. The asymptotic energy spectrum is computed by solving the Bethe-Yang equations which determine the allowed values of the momenta compatible with the periodic boundary conditions.

The leading order exponential corrections to the energy of the ground state, known as wrapping corrections, come from virtual particles wrapping the space circle. To compute the energy spectrum at finite volume $R$, one should evaluate the sum over all possible wrapping processes. An efficient and elegant way to do that, suggested by Alexey Zamolodchikov [1-3], is to compactify also the time circle at asymptotically large distance $L$ and formulate the problem in the mirror channel where the space and the time are interchanged. 
Then the sum over the virtual wrapping particles, which become real particles in the mirror channel, can be performed with the techniques of the Thermodynamic Bethe Ansatz (TBA) [4]. The sum is saturated by a saddle point determined by a set of non-linear integral equations for the particle densities known as TBA equations. This strategy works also in the AdS/CFT integrability [5], where there is no explicit Lorentz invariance. In this case the dynamics in the physical and in the mirror channels look differently and one speaks of physical and mirror theories.

Although the TBA equations are derived for the thermodynamical state involving infinite number of particles, they are able to reproduce the Lüscher corrections [6] in the limit of asymptotically large $R$ where the wrapping particles are scarce and the thermodynamical arguments are not justified. This suggests that the TBA equations are in fact exact identities for some statistical theory describing the grand canonical ensemble of virtual particles in the mirror channel. Such a statistical description, which technically represents an exact cluster expansion, was obtained by several authors in [7-12]. The latter proved to be a useful tool to solve some concrete problems $[13,14]$.

Another possible strategy to go beyond the thermodynamics is to consider the particle densities as fluctuating fields and evaluate the quantum corrections to the TBA equation. Woynarovich [15] found that the gaussian fluctuations lead to a non-extensive $O(1)$ term in the free energy. Pozsgay [16] then pointed out that in case of periodic boundary conditions second subleading contribution coming from the Gaudin measure which cancels the first one. Very recently Jiang, Komatsu and Vescovi [17] proposed a path-integral formulation of the TBA partition function based on an action which, importantly, differs from the Yang-Yang potential, the most natural candidate for such an action. ${ }^{1}$ The path integral proposed in [17] does generate an $O(1)$ term through the gaussian fluctuations, but this can be easily cured by introducing an extra fermionic field which generates the Gaudin measure.

As a matter of fact, it is straightforward to formulate an effective QFT having as perturbative series the exact cluster expansion for TBA in the form given in $[11,12]$. The idea for such a construction is contained in the field-theoretical proof of the matrix-tree theorem given in the appendis B of [12]. This QFT involves a pair of bosons as well as their fermionic partners and the bosonic part of the action matches the action proposed in [17].

In this article we adress the inverse problem, namely to construct from first principles a conceptually satisfactory effective QFT whose perturbative description is given by the exact cluster expansion. To explain the construction in a simple setup, we consider a scattering theory for a single species of neutral massive particles and no bound states. For such a theory there are no Bethe strings and all excitations have real rapidities. The only example of such a theory is given by the sinh-Gordon model, but in view of possible applications to AdS/CFT, we do not assume relativistic invariance and the scattering matrix being of difference type. We only assume that the scattering phases in the mirror channel are obtained by analytical continuation of the scattering phases in the physical channel.

\footnotetext{
${ }^{1}$ The Yang-Yang potential was introduced in [4] as a formal tool to prove the existence of the solutions of Bethe equations by variational principle. In the last decade it attracted the attention with its connection, discovered by Nekrasov and Shatashvili [18], to the instanton counting of four-dimensional gauge theories.
} 
We are not going to construct explicitly the wave functions for periodic boundary conditions and in this respect our approach has no significant overlap with the field-theoretical derivation of the TBA equations using the form factor bootstrap discussed in [19]. Instead we will postulate that if the scattering theory is confined in a finite but asymptotically large volume, there exists, both in the physical and in the mirror theories, an over-complete set of normalised multi-particle states with unrestricted rapidities. Two distinct states are orthogonal if both sets of rapidities are on-shell, i.e. if they solve the Bethe-Yang equations. If the field theory can be constructed as a continuum limit of a spin chain, which is so in most of the interesting cases [5, 20-27], these states are the off-shell Bethe states of the Algebraic Bethe Ansatz.

The paper is organised as follows. In section 2 we introduce the Hilbert space and the operator content of the effective QFT. The Hilbert space is generated by two families of operators which we call wrapping operators because they create particles wrapping the main cycles of the torus. The wrapping operators associated with the same cycle strictly commute while the operators associated with two intersecting cycles commute by the scattering matrix. The modular invariance leads to operator constraints which are equivalent to the Bethe-Yang equations. The wrapping operators are represented as vertex operators acting in the Fock space of a pair of bosonic fields. The two bosonic fields are the operators for the scattering phases of a mirror particle wrapping the spatial or the temporal cycles.

In section 3 we represent the partition function on a torus with one of its periods asymptotically large as the expectation value in the Fock space of the effective QFT. We use the operator formulation of the Bethe-Yang equations to transform the sum over on-shell states into a contour integral. The integration measure involves the Gaudin determinant, which is taken into account by an extra pair of fermionic fields.

In section 4 we formulate the path-integral formultaion for the effective QFT is obtained by introducing two extra pairs of Hubbard-Stratonovich fields which generate respectively the bosonic and the fermionic correlators. We show that the path integral possesses a fermionic symmetry which gets it localised to the critical point of the action and renders the theory one-loop exact. For periodic boundary conditions the one-loop effects compensate completely.

In section 5 we consider the example of the sinh-Gordon model, where we show that the critical point of action is determined by the discrete Liouville equation. We conclude with section 6. Appendix A contains our conventions for the scattering phases in the physical and in the mirror channels. In appendix B we derive the operator representation of the Gaudin determinant.

\section{Degrees of freedom of the effective QFT}

\subsection{Space and time wrapping operators}

The effective QFT involves a minimal subset of the degrees of freedom of the original field theory after the latter is compactified on a torus with asymptotically large space and time circles. The Fock space of the effective QFT is generated by two families of operators, 
$\mathbf{A}(u)$ and $\mathbf{B}(u)$, associated with the two homotopy cycles, $A$ and $B$, of the space-time torus. Here we adopt the following convention for the two cycles,

$$
\begin{aligned}
A \text {-cycle } & =\text { temporal circle in the physical kinematics } \\
& =\text { spatial circle in the mirror kinematics } \\
B \text {-cycle } & =\text { spatial circle in the physical kinematics } \\
& =\text { temporal circle in the mirror kinematics. }
\end{aligned}
$$

To avoid confusion let us stress that the Fock space we construct is not directly related to the Hilbert spaces of the physical or mirror theories. In particular, the mirror transformation is an automorphism of the Fock space.

The wrapping particles will be represented as non-contractible loops winding around the two cycles of the torus as shown in figure 1. This representation is natural in the microscopic picture based on a spin chain. When two lines cross, there is an additional scattering phase factor. Both types of wrapping particles can be defined off-shell and their rapidities can take any complex values. The $M$-particle off-shell states are labeled by the non-ordered set of rapidities $\left\{u_{1}, \ldots, u_{M}\right\}$ and are assumed to be normalisable but not necessarily orthogonal. They are eigenstates of the Hamiltonian with eigenvalues given by the sum of the energies of the constituent particles. The on-shell states are singled out by the periodicity conditions on the rapidities and belong to a discrete spectrum.

Let us denote by $\mathbf{A}(u)$ the operator creating a particle of rapidity $u$ in the physical kinematics wrapping the $A$-cycle. Similarly, denote by $\mathbf{B}(u)$ the operator creating a particle of rapidity $u$ in the mirror kinematics wrapping the $B$-cycle. The operators $\mathbf{A}$ with real rapidities create real particles in the physical channel or virtual particles in the mirror channel. Conversely, the operators $\mathbf{B}$ with real rapidities create real particles in the mirror channel or virtual particles in the physical channel.

An operator defined in the physical theory can be transformed by analytic continuation $u \rightarrow u^{ \pm \gamma}$ into an operator defined in the mirror theory and vice versa. ${ }^{2}$ The inverse transformation will be denoted by $u^{-\gamma}$. For the sake of clarity we give the list the four types of operators,

$$
\begin{array}{ll}
\mathbf{B}(u) & - \text { creates a mirror particle wrapping the } B \text {-cycle, } \\
\mathbf{A}\left(u^{\gamma}\right) & - \text { creates a mirror particle wrapping the } A \text {-cycle } \\
\mathbf{B}\left(u^{-\gamma}\right) & - \text { creates a physical particle wrapping the } B \text {-cycle. } \\
\mathbf{A}(u) & - \text { creates a physical particle wrapping the } A \text {-cycle, }
\end{array}
$$

The wrapping operators are holomorphic functions of the non-restricted rapidity variable $u$ and their analytic properties are determined by those of the S-matrix. Since the off-shell states are by construction symmetric under permutations, the operators creating particles wrapping the same cycle commute. Morover, the expectation values of products

\footnotetext{
${ }^{2}$ Our conventions for the mirror transformation are summarised in appendix A. Our notations are conform with those used in AdS/CFT integrability.
} 

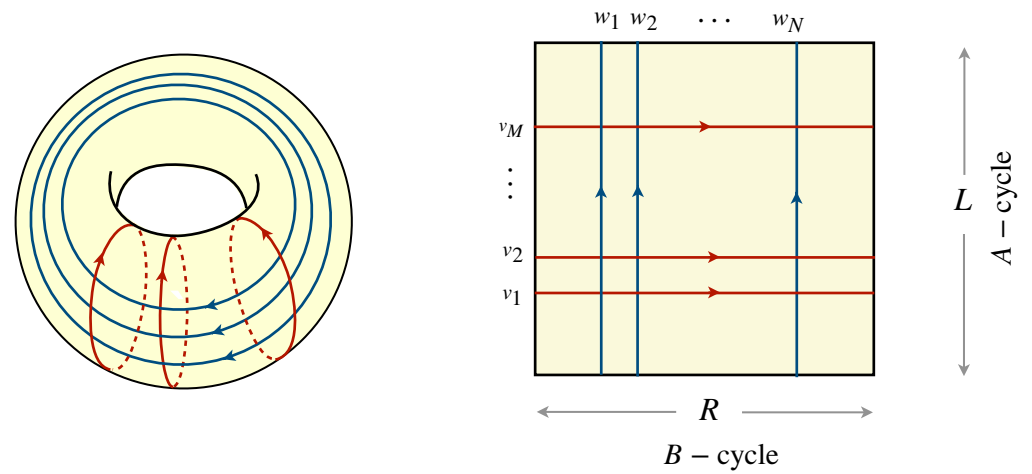

Figure 1. Particles wrapping the space and the time circles. On the left the wrapping particles are depicted as closed paths winding around the two main circles of the torus. On the right the wrapping particles are represented by vertical and horizontal lines on a periodic rectangle. The topology requires that each pair of a space and a time wrapping particles scatter once.

of wrapping operators of the same kind factorise,

$$
\begin{gathered}
\left\langle\prod_{j=1}^{M} \mathbf{B}\left(v_{j}\right)\right\rangle_{L, R}=\frac{\left\langle v_{1}, \ldots, v_{M}\left|e^{-R \tilde{\mathbf{H}}}\right| v_{1}, \ldots, v_{M}\right\rangle}{\left\langle v_{1}, \ldots, v_{M} \mid v_{1}, \ldots, v_{M}\right\rangle}=\prod_{j=1}^{M} e^{-R \tilde{E}\left(v_{j}\right)}, \\
\left\langle\prod_{k=1}^{N} \mathbf{A}\left(w_{j}\right)\right\rangle_{L, R}=\frac{\left\langle w_{1}, \ldots, w_{M}\left|e^{-L \mathbf{H}}\right| w_{1}, \ldots, w_{M}\right\rangle}{\left\langle w_{1}, \ldots, w_{M} \mid w_{1}, \ldots, w_{M}\right\rangle}=\prod_{k=1}^{N} e^{-L E\left(w_{j}\right)} .
\end{gathered}
$$

The expectation values for the virtual wrapping particles are related to those for the real particles by analytic continuation,

$$
\left\langle\prod_{j=1}^{M} \mathbf{B}\left(u_{j}^{-\gamma}\right)\right\rangle_{L, R}=\prod_{j=1}^{M} e^{i R p\left(u_{j}\right)}, \quad\left\langle\prod_{k=1}^{N} \mathbf{A}\left(u_{k}^{\gamma}\right)\right\rangle_{L, R}=\prod_{k=1}^{N} e^{-i L \tilde{p}\left(u_{k}\right)} .
$$

Now let us consider the expectation value involving both types of wrapping operators. For an observer positioned in the physical channel, the expectation value of $N$ real and $M$ virtual particles, illustrated by figure 1, is equal to the product of their Boltzmann factors and the scattering factors between the real and virtual particles,

$$
\left\langle\prod_{j=1}^{M} \mathbf{B}\left(v_{j}\right) \prod_{k=1}^{N} \mathbf{A}\left(w_{k}\right)\right\rangle_{L, R}=\prod_{j=1}^{M} \prod_{k=1}^{N} S\left(v_{j}^{\gamma}, w_{k}\right) \prod_{j=1}^{M} e^{-\tilde{E}\left(v_{j}\right)} \prod_{k=1}^{N} e^{-L E\left(w_{k}\right)} .
$$

There is an obvious Fock-space representation of the wrapping operators and the expectation value (2.5). Assume that the two families of operators satisfy the algebra

$$
\mathbf{B}(v) \mathbf{A}(w)=S\left(v^{\gamma}, w\right) \mathbf{A}(w) \mathbf{B}(v), \quad[\mathbf{A}(u), \mathbf{A}(v)]=[\mathbf{B}(u), \mathbf{B}(v)]=0
$$

and introduce the vacuum state $|R\rangle$ and the dual vacuum state $\langle L|$ so that

$$
\left\langle L\left|\mathbf{A}(u)=e^{-L E(u)}\langle L|, \quad \mathbf{B}(u)| R\rangle=e^{-R \tilde{E}(u)}\right| R\right\rangle .
$$


With the convention $\langle L \mid R\rangle=1$, the expectation value (2.5) is represented by the scalar product

$$
\left\langle\prod_{j=1}^{M} \mathbf{B}\left(v_{j}\right) \prod_{k=1}^{N} \mathbf{A}\left(w_{k}\right)\right\rangle_{L, R} \stackrel{\text { def }}{=}\left\langle L\left|\prod_{j=1}^{M} \mathbf{B}\left(v_{j}\right) \prod_{k=1}^{N} \mathbf{A}\left(w_{k}\right)\right| R\right\rangle .
$$

It is convenient to include in the definition of the Fock-space expectation value the anti-normal ordering $\vdots$ : which automatically puts all operators $\mathbf{B}$ on the left of all operators $\mathbf{A}$,

$$
\langle\mathcal{O}\rangle_{L, R} \equiv\langle L|: \mathcal{O}:| R\rangle
$$

With this definition the order of the operators inside the brackets does not matter.

\subsection{Operator form of the Bethe-Yang equations}

We would like to sum over the on-shell states without actually solving the Bethe-Yang equations. This can be achieved by reformulating the on-shell conditions on the rapidities as operator constraints.

The quantisation of the rapidities in the mirror channel is imposed by the periodicity conditions, which express the invariance of the expectation value under replacing the cycles $A$ and $B$ by $A$ and $B+A$, applied separately for each particle. In terms of wrapping operators this signifies that the expectation value is invariant under replacing $\mathbf{B}\left(v_{l}\right)$ by $-\mathbf{A}\left(v_{l}^{\gamma}\right) \mathbf{B}\left(v_{l}\right)$,

$$
\left\langle\left(1+\mathbf{A}\left(v_{l}^{\gamma}\right)\right) \prod_{j=1}^{M} \mathbf{B}\left(v_{j}\right) \prod_{k=1}^{N} \mathbf{A}\left(w_{k}\right)\right\rangle_{L, R}=0, \quad l=1, \ldots, M .
$$

The minus sign comes from $\left\langle\mathbf{A}\left(v_{l}^{\gamma}\right) \mathbf{B}\left(v_{l}\right)\right\rangle_{L, R}=-1$. Equation (2.10) is equivalent to the Bethe-Yang equations in the mirror channel,

$$
e^{-i \tilde{\phi}_{l}} \equiv e^{-i L \tilde{p}\left(v_{l}\right)} \prod_{j=1}^{M} \tilde{S}\left(v_{j}, v_{l}\right)=-1, \quad l=1, \ldots, M
$$

where $\tilde{S}$ denotes the S-matrix in the mirror channel,

$$
\tilde{S}(u, v) \equiv S\left(u^{\gamma}, v^{\gamma}\right)
$$

Intuitively, the insertion of the operator $-\mathbf{A}\left(v_{l}^{\gamma}\right)$ has the effecy of adding an extra piece to the world line of $l$-th mirror particle: the particle travels around the mirror space before wrapping the mirror time circle, as shown in figure 2.

In a similar way, the invariance under replacing the cycles $A$ and $B$ by $A+B$ and $B$ leads to the equations

$$
\left\langle\left(1+\mathbf{B}\left(w_{l}^{-\gamma}\right)\right) \prod_{j=1}^{M} \mathbf{B}\left(v_{j}\right) \prod_{k=1}^{N} \mathbf{A}\left(w_{k}\right)\right\rangle_{L, R}=0, \quad l=1, \ldots, N
$$




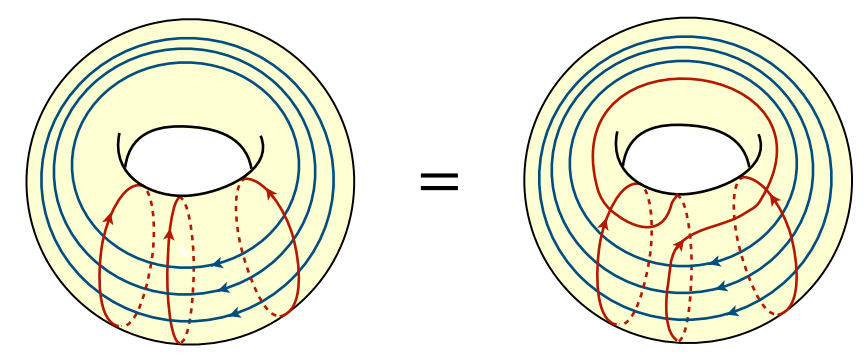

Figure 2. Pictorial representation of the quantisation condition (2.10).

which imposes $N$ constraints for the rapidities of the physical particles (the Bethe-Yang equatiins in the physical channel)

$$
e^{i \varphi_{l}} \equiv e^{i R p\left(w_{l}\right)} \prod_{k=1}^{N} S\left(w_{l}, w_{k}\right)=-1, \quad l=1, \ldots, N .
$$

\subsection{Free-field realisation}

The wrapping operators admit a representation as vertex operators for free fields, similar to for the ZF operators [28]. Since we are interested in computing the trace in the mirror channel, we associate the free fields with the wrapping operators in mirror kinematics, $\mathbf{B}(u)$ and $\mathbf{A}\left(u^{\gamma}\right)$,

$$
\mathbf{B}(u)=e^{-\varphi(u)}, \quad \mathbf{A}\left(u^{\gamma}\right)=e^{-i \bar{\varphi}(u)} .
$$

The algebra (2.6) is satisfied if the two fields have commutation relations ${ }^{3,4}$

$$
[\boldsymbol{\varphi}(u), \bar{\varphi}(v)]=i \log \tilde{S}(u, v), \quad[\overline{\boldsymbol{\varphi}}(u), \overline{\boldsymbol{\varphi}}(v)]=[\boldsymbol{\varphi}(u), \boldsymbol{\varphi}(v)]=0 .
$$

The action of the two bosonic fields on the Fock vacua is determined by (2.7).

$$
\left\langle L\left|\bar{\varphi}(u)=\bar{\varphi}^{\circ}(u)\langle L|, \quad \varphi(u)| R\rangle=\varphi^{\circ}(u)\right| R\right\rangle,
$$

where we introduced notations for the classical values $\bar{\varphi}^{\circ}$ and $\varphi^{\circ}$ of the two fields,

$$
\varphi^{\circ}(u)=R \tilde{E}(u), \quad \bar{\varphi}^{\circ}(u)=L \tilde{p}(u) .
$$

More generally the classical value $\varphi^{\circ}$ can be chosen to be any linear combination of conserved charges densities $\tilde{h}_{n}(u)$ in the mirror theory,

$$
\varphi^{\circ}(u)=\sum_{n} t_{n} \tilde{h}_{n}(u)
$$

with $t_{1}=R$ and $\tilde{h}_{1}(u)=\tilde{E}(u)$. The expectation value of operators built from the bosonic fields is defined again by $(2.9)$, where the anti-normal ordering $\vdots \vdots$ means that all operators $\varphi$ should be put on the left of all operators $\bar{\varphi}$.

\footnotetext{
${ }^{3}$ Instead of two fields one can speak of the positive and the negative frequency parts of the same bosonic field. This can be done once the concrete form of the scattering matrix is known.

${ }^{4}$ The branch of the logarithm can be determined by so that the scattering phase vanishes if one of the rapidities is sent to indinity.
} 
In terms of the gaussian field the on-shell condition for the mirror $M$-particle states takes the form

$$
\left\langle\left(1+e^{-i \bar{\varphi}\left(v_{l}\right)}\right) \prod_{j=1}^{M} e^{-\varphi\left(v_{j}\right)}\right\rangle_{L, R}=0, \quad l=1, \ldots, M .
$$

\section{Partition function on the torus}

\subsection{The partition function as a sum over on-shell states}

The partition function on the torus can be expressed as a thermal trace of the evolution operator either in the Hilbert space $\mathcal{H}$ in the physical channel or in the Hilbert space $\tilde{\mathcal{H}}$ in the mirror channel,

$$
\mathcal{Z}(L, R)=\operatorname{Tr}_{\mathcal{H}} e^{-L \mathbf{H}_{R}}=\operatorname{Tr}_{\tilde{\mathcal{H}}} e^{-R \tilde{\mathbf{H}}_{L}} .
$$

The first representation is also called $L$-picture while the second representation is called $R$-picture. When $L$ is asymptotically large and $R$ is finite, the sum in the $R$-picture is dominated by the ground state contribution since the relative contribution of the excited states is exponentially small. The trace in the $R$-picture is not accessible for calculation because the spectrum at finite $R$ is not known. On the other hand, in the $L$ picture we can replace the hamiltonian $\tilde{\mathbf{H}}_{L}$ by $\tilde{\mathbf{H}} \equiv \tilde{\mathbf{H}}_{\infty}$ whose spectrum is given by the Bethe-Yang equations.

The $M$-particle on-shell states in the mirror channel are characterised by the semiinteger numbers $n_{1}<n_{2}<\ldots<n_{M}$, called Bethe numbers, which arise in the logarithmic form of the Bethe-Yang equations

$$
\tilde{\phi}_{l}\left(u_{1}, \ldots, u_{M}\right) \equiv\left\langle\bar{\varphi}\left(v_{l}\right) \prod_{j=1}^{M} e^{-\varphi\left(v_{j}\right)}\right\rangle=-2 \pi n_{j}, \quad j=1, \ldots, M .
$$

The partition function in the $L$-picture is given by the sum over all Bethe numbers

$$
\mathcal{Z}(L, R)=\sum_{M=0}^{\infty} \sum_{n_{1}<\ldots<n_{M}} \prod_{j=1}^{M} e^{-R \tilde{E}\left(v_{j}\right)},
$$

with $\mathbf{v}=\left\{v_{1}, \ldots, v_{M}\right\}$ given implicitly as the solution of (3.2). The Bethe numbers should be all different because of the "fermionic" property (A.5) of the S-matrix.

We would like to write this series as a Fock space expectation value. For that we need first to get rid of the condition that all rapidities in the sum in (3.3) are different. This condition can be relaxed by introducing "non-physical" solutions of the Bethe-Yang equations (3.2) such that the rapidities of two or more particles can coincide [8-11, 19, 29]. A stack of $r$ identical Bethe roots $u$ will be denoted by $u^{r}$ and $r$ will be called multiplicity. The generalised $M$-particle states are characterised by the rapidities and their multiplicities $\mathbf{v}^{\mathbf{r}}=\left\{v_{1}^{r_{1}}, \ldots, v_{m}^{r_{m}}\right\}$, where $M=r_{1}+\ldots+r_{m}$. The logarithmic form of the Bethe-Yang equations for such states is

$$
\begin{aligned}
& \tilde{\phi}_{l}=-2 \pi n_{l}, \quad l=1, \ldots, m \\
& \tilde{\phi}_{l} \equiv\left\langle\bar{\varphi}\left(v_{l}\right) \prod_{j=1}^{m} e^{-r_{j} \varphi\left(v_{j}\right)}\right\rangle=\tilde{p}\left(u_{l}\right) L+\frac{1}{i} \sum_{k(\neq l)}^{m} r_{k} \log \tilde{S}\left(u_{l}, u_{k}\right)+\pi\left(r_{l}-1\right) .
\end{aligned}
$$




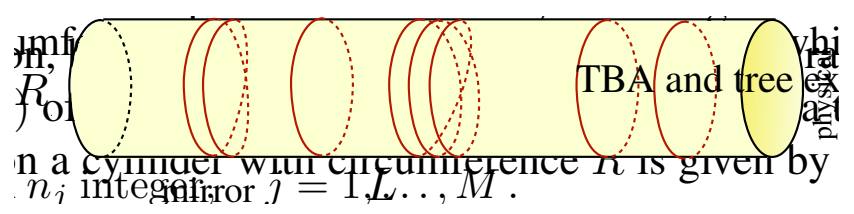

Figure 3. Virtual particles wrapping the space multiple times.

Now the sum over "repulsing" Bethe numbers can be expressed as a non-restricted sum of mode numbers and their multiplicities

$$
\begin{aligned}
\mathcal{Z}(L, R) & =\sum_{M=0}^{\infty} \frac{1}{M !} \sum_{n_{1}, \ldots, n_{M}} \prod_{j<k}^{M}\left(1-\delta_{n_{j}, n_{k}}\right) \prod_{j=1}^{M} e^{-R \tilde{E}\left(u_{j}\right)} \\
& =\sum_{m=0}^{\infty} \frac{1}{m !} \sum_{r_{1}, \ldots, r_{m} \geq 1} \sum_{n_{1}, \ldots, n_{m}} \prod_{j=1}^{m} \frac{(-1)^{r_{j}-1}}{r_{j}} e^{-r_{j} R \tilde{E}\left(u_{j}\right)} .
\end{aligned}
$$

The last line is obtained by expanding the product of Kronecker symbols.

A Bethe root of multiplicity $r$ can be interpreted as a single mirror particle wrapping $r$ times the $R$-cycle as shown in figure 3 . The operator creating such a particle is

$$
\underbrace{\left.\left(\int\right)^{\prime}\right)}_{r}=\frac{(-1)^{r-1}}{r} e^{-r \varphi(u)} .
$$

An $r$-wrapping particle scatters with itself $r-1$ times, which yields a factor $[\tilde{S}(u, u)]^{r-1}=$ $(-1)^{r-1}$. The combinatorial factor $1 / r$ counts for the $\mathbb{Z}_{r}$ cyclic symmetry of the $r$-wrapping loop.

\subsection{Rewriting the sum as a contour integral}

Our goal is to perform the sum over the on-shell states without solving the Bethe-Yang equations. This can be achieved by transforming the sum over the Bethe numbers in eq. (3.5) into a multiple contour integral. Let us first consider the sector $\tilde{\mathcal{H}}_{L, \mathbf{r}}$ of the Hilbert space $\tilde{\mathcal{H}}$ in the mirror channel, spanned by all generalised on-shell states of the form $\mathbf{u}^{\mathbf{r}}=\left\{u_{1}^{r_{1}}, \ldots, u_{m}^{r_{m}}\right\}$ with fixed wrapping numbers $\mathbf{r}=\left\{r_{1}, \ldots, r_{m}\right\}$. The on-shell states belonging to $\tilde{\mathcal{H}}_{L, \mathbf{r}}$ are characterised by $m$ unrestricted Bethe numbers $n_{1}, \ldots, n_{m}$.

The thermal trace restricted to this sector can be expressed as an $m$-fold contour integral involving the $m$ scattering phases defined by (3.4),

$$
\begin{aligned}
\operatorname{Tr}_{\tilde{\mathcal{H}}_{L, \mathbf{r}}} e^{-R \tilde{\mathbf{H}}_{L}} & =\sum_{n_{1}, \ldots, n_{m}} \prod_{j=1}^{m} e^{-r_{j} R \tilde{E}\left(u_{j}\right)} \\
& =\oint_{\mathbb{R}^{m}} \prod_{j=1}^{m} \frac{d \log \left[1+e^{-i \tilde{\phi}_{j}}\right]}{2 \pi i} e^{-r_{j} R \tilde{E}\left(u_{j}\right)}
\end{aligned}
$$


with each contour of integration encircling the real axis anticlockwise. Expanding the differentials we write, more explicitly,

$$
\text { r.h.s. of }(3.7)=(-i)^{N} \oint_{\mathbb{R}^{m}} \prod_{j=1}^{m} \frac{d u_{j}}{2 \pi i} \frac{e^{-r_{j} R \tilde{E}\left(u_{j}\right)}}{1+e^{i \tilde{\phi}_{j}}} \tilde{G}\left(\mathbf{u}^{\mathbf{r}}\right),
$$

where the last factor is the Jacobian of the change of variables from the phases to the rapidities known also as Gaudin determinant,

$$
\tilde{G}\left(\mathbf{u}^{\mathbf{r}}\right)=\operatorname{det} \tilde{G}_{k j}, \quad \tilde{G}_{k j}=\frac{\partial \tilde{\phi}_{j}\left(\mathbf{u}^{\mathbf{r}}\right)}{\partial u_{k}} .
$$

Now we are going to represent the integrand in (3.8) as a Fock-space expectation value. For that we have to give an operator representation of the Gaudin determinant. As we show in appendix B, this can be achieved by introducing extra fermionic fields $\bar{\psi}(u)$ and $\boldsymbol{\psi}(u)$ defined by

$$
\begin{aligned}
{[\boldsymbol{\psi}(u), \overline{\boldsymbol{\psi}}(v)]_{+} } & =-i \log \tilde{S}(u, v), & {[\overline{\boldsymbol{\psi}}(u), \overline{\boldsymbol{\psi}}(v)] } & =[\boldsymbol{\psi}(u), \boldsymbol{\psi}(v)]=0 . \\
\langle L| \overline{\boldsymbol{\psi}}(u) & =0, & \boldsymbol{\psi}(u)|R\rangle & =0 .
\end{aligned}
$$

The expectation value \langle\rangle$_{L, R}$ is extended to operators involving fermionic fields again by the anti-normal product convention. The fermionic formula for the Gaudin determinant, derived in appendix B, which will be the basic technical tool for constructing the effective QFT, reads,

$$
\tilde{G}\left(\mathbf{u}^{\mathbf{r}}\right) \prod_{j=1}^{m} e^{-R r_{j} \tilde{E}\left(u_{j}\right)}=\left\langle\prod_{j=1}^{m}\left[\overline{\boldsymbol{\varphi}}^{\prime}\left(u_{j}\right)-r_{j} \overline{\boldsymbol{\psi}}\left(u_{j}\right) \boldsymbol{\psi}^{\prime}\left(u_{j}\right)\right] e^{-r_{j} \boldsymbol{\varphi}\left(u_{j}\right)}\right\rangle_{L, R} .
$$

With the help of the fermionic formula we write the r.h.s. of (3.7) as

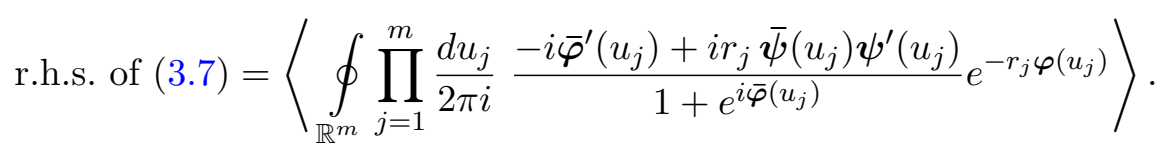

Performing the sum over the multipliticites in (3.11) with the appropriate weights, we obtain the desired Fock-space representation of the partition function,

$$
\mathcal{Z}(L, R)=\langle\hat{\mathbf{\Omega}}\rangle_{L, R}
$$

with the operator $\hat{\boldsymbol{\Omega}}$ defined by

$$
\begin{aligned}
\hat{\boldsymbol{\Omega}} & \equiv \exp \oint_{\mathbb{R}} \frac{d u}{2 \pi i}\left(-i \overline{\boldsymbol{\varphi}}^{\prime}(u) \log \left(1+e^{-\boldsymbol{\varphi}(u)}\right)+\frac{i \overline{\boldsymbol{\psi}}(u) \boldsymbol{\psi}^{\prime}(u)}{1+e^{\boldsymbol{\varphi}(u)}}\right) \frac{1}{1+e^{i \overline{\boldsymbol{\varphi}}(u)}} \\
& =\exp \oint_{\mathbb{R}} \frac{d u}{2 \pi i}\left[\log \left(1+e^{-\boldsymbol{\varphi}(u)}\right) \partial_{u} \log \left(1+e^{-i \overline{\boldsymbol{\varphi}}(u)}\right)+\frac{i \overline{\boldsymbol{\psi}}(u) \boldsymbol{\psi}^{\prime}(u)}{\left(1+e^{\boldsymbol{\varphi}(u)}\right)\left(1+e^{i \overline{\boldsymbol{\varphi}}(u)}\right)}\right],
\end{aligned}
$$

the contour of integration encircling the real axis anticlockwise.

Remarkably, the integrand is symmetric with respect of exchanging $i \bar{\varphi}$ and $\boldsymbol{\varphi}$. The asymmetry is introduced by the contour of integration which encircles the zeroes of $1+e^{i \bar{\varphi}}$ only and not those of $1+e^{\varphi}$. 


\subsection{Continuous spectrum approximation}

The operator representation (3.13) is justified only when the bare expectation value $\bar{\varphi}^{\circ}=$ $\langle\bar{\varphi}\rangle$ is asymptotically large, $\bar{\varphi}^{\circ}=L \tilde{p}(u)$. Then one can replace in (3.14) with exponential accuracy

$$
\log \left(1+e^{-i \bar{\varphi}(u)}\right) \rightarrow\left\{\begin{array}{cc}
-i \bar{\varphi}(u) & \text { if } \Im u>0 \\
0 & \text { if } \Im u<0
\end{array}\right.
$$

and

$$
\frac{1}{1+e^{i \bar{\varphi}(u)}} \rightarrow \begin{cases}1 & \text { if } \Im u>0 \\ 0 & \text { if } \Im u<0 .\end{cases}
$$

Hence the contour integral in (3.14) can be approximated by a linear integral slightly above the real axis, with an extra minus sign because of the orientation of the contour, and the operator representation (3.13) simplifies to

$$
\mathcal{Z}(L, R)=\langle\check{\boldsymbol{\Omega}}\rangle_{L, R}, \quad \check{\boldsymbol{\Omega}} \equiv \exp \int_{-\infty}^{\infty} \frac{d u}{2 \pi}\left[\log \left(1+e^{-\boldsymbol{\varphi}(u)}\right) \overline{\boldsymbol{\varphi}}^{\prime}(u)-\frac{\overline{\boldsymbol{\psi}}(u) \boldsymbol{\psi}^{\prime}(u)}{1+e^{\boldsymbol{\varphi}(u)}}\right]
$$

The expectation value in (3.17) generates the sum over all solutions of the Bethe-Yang equations. Since the scattering phases are of order of $L$, the rapidities are spaced at $\sim 1 / L$ and the sum can be approximated by an integral up to exponentially small in $L$ corrections.

The graphs in the combinatorial approach of [11] appear as Feynman diagrams when the expectation value in (3.17) is evaluated perturbatively starting with the classical values $\bar{\varphi}^{\circ}$ and $\varphi^{\circ}$. Denoting by $\varphi_{q}$ and $\bar{\varphi}_{q}$ the quantum part of the bosonic fields,

$$
\varphi(u)=\varphi^{\circ}(u)+\varphi_{q}(u), \quad \bar{\varphi}(u)=\bar{\varphi}^{\circ}(u)+\bar{\varphi}_{q}(u),
$$

the Feynman graphs are composed of vertices generated by the expansion of the nonpolynomial potential $\log \left(1+e^{-\varphi^{\circ}-\varphi_{q}}\right)$, and propagators given by

$$
\left\langle\overline{\boldsymbol{\varphi}}_{q}^{\prime}(u) \boldsymbol{\varphi}_{q}(v)\right\rangle_{L, R}=-\tilde{K}(u, v), \quad\left\langle\boldsymbol{\psi}^{\prime}(u) \overline{\boldsymbol{\psi}}(v)\right\rangle_{L, R}=\tilde{K}(u, v)
$$

where $\tilde{K}$ is the scattering kernel in the mirror theory

$$
\tilde{K}(u, v)=\frac{1}{i} \partial_{u} \log \tilde{S}(u, v) .
$$

The propagators are in general not symmetric and should be represented by oriented lines.

As in any perturbative QFT, the free energy $\mathcal{F}(L, R)=\log \mathcal{Z}(L, R)$ is equal to the sum of all connected Feynman graphs, which are either trees or trees with one cycle. For periodic boundary conditions the fermionic and the bosonic cycles compensate each other and only the trees contribute to the free energy. These trees describe clusters of wrapping particles sketched in figure 4. The generating function for the trees satisfies a non-linear integral equation, which is nothing but the TBA equation for a theory with diagonal scattering [11]. 


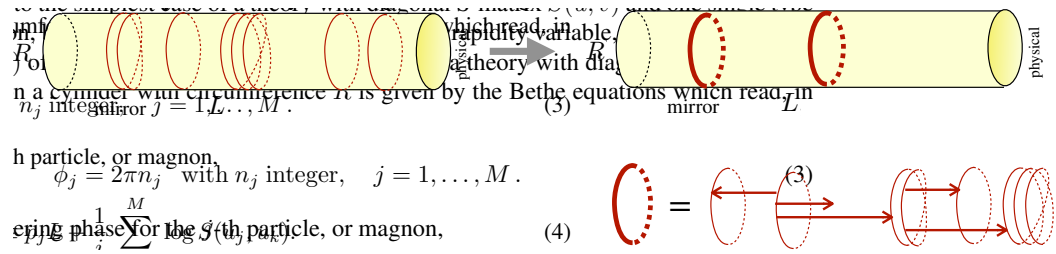

Figure 4. Wrapping particles put in a large box of length $L$ form a non-ideal gas with interaction $1 / L$. The free energy is a sum over non-interacting clusters of wrapping particles. The perturbative series of the expectation value (3.17) gives the exact cluster expansion to all orders.

\subsection{Excited states in the physical channel}

Let us consider the more interesing case of an excited state with rapidities $\mathbf{w}=$ $\left\{w_{1}, \ldots, . w_{M}\right\}$ in the physical channel. The excited states in finite volume have the same particle structure as in the asymptotically large volume, but the rapidities of the particles satisfy the so called "exact Bethe equations", which take into account the wrapping effects.

The excited state partition function is obtained by performing in the expectation value (2.8) the sum over all on-shell rapidities v. Equivalently, one can insert in the expectation value in (3.17) $N$ physical wrapping operators with rapidities $\mathbf{w}=\left\{w_{1}, \ldots, . w_{M}\right\}$,

$$
\mathcal{Z}(L, R, \mathbf{w})=\left\langle\prod_{j=1}^{N} \mathbf{A}\left(w_{j}\right) \check{\boldsymbol{\Omega}}\right\rangle_{L, R}, \quad \mathbf{A}\left(w^{\gamma}\right) \equiv \exp [-i \overline{\boldsymbol{\varphi}}(w)] .
$$

The rapidities $\mathbf{w}$ must solve the exact Bethe equations in the physical channel, which is equivalent to imposing the operator constraint

$$
\left\langle\left(1+\mathbf{B}\left(w_{l}^{-\gamma}\right)\right) \prod_{j=1}^{N} \mathbf{A}\left(w_{j}\right) \check{\boldsymbol{\Omega}}\right\rangle_{L, R}=0, \quad l=1, \ldots, N,
$$

with $\mathbf{B}(w) \equiv e^{-\varphi(w)}$.

The excited state contributes to the free energy directly by the energies of the $N$ particles as well as indirectly by modifying the Bethe-Yang equations for the rapidities of the virtual particles in the mirror channel. This is taken into account automatically by the expectation value. On the other hand, the mirror wrapping particles from the thermal ensemble backreact by deforming the quantisation condition for the rapidities $\mathbf{w}$. Therefore if we first compute the partition function (3.21) and then determine the exact Bethe roots $\mathbf{w}$ from (3.22), the result may be incorrect.

The proper way to introduce the excited state is as a contour integral which automatically imposes the on-shell condition (3.22). That is, the product of operators inserted in the expectation value (3.21) should be replaced by a multiple contour integral around the exact Bethe roots. The integrand of the $N$-fold contour integral contains a Jacobian which can be expressed in terms of the free fermions as in (3.12), but with $\varphi$ and $i \bar{\varphi}$ interchanged. This results in replacing in the expectation value (3.21)

$$
\prod_{j=1}^{N} e^{-i \overline{\boldsymbol{\varphi}}\left(w_{j}^{-\gamma}\right)} \rightarrow \frac{(-1)^{N}}{N !} \oint_{\mathbf{w}^{-\gamma}} \prod_{j=1}^{N} \frac{d u_{j}}{2 \pi i} e^{-i \overline{\boldsymbol{\varphi}}\left(u_{j}\right)} \frac{\boldsymbol{\varphi}^{\prime}\left(u_{j}\right)-i \overline{\boldsymbol{\psi}}\left(u_{j}\right) \boldsymbol{\psi}^{\prime}\left(u_{j}\right)}{1+e^{\boldsymbol{\varphi}\left(u_{j}\right)}}
$$


However there is another, more elegant way to impose the on-shell condition, namely by lifting the on-shell condition in the exponent, which allows to write it as a single contour integral,

$$
\prod_{j=1}^{N} e^{-i \overline{\boldsymbol{\varphi}}\left(w_{j}^{-\gamma}\right)} \rightarrow \exp \left[\oint_{\mathbf{w}^{-\gamma}} \frac{d u}{2 \pi i}(-i \overline{\boldsymbol{\varphi}}(u)) \frac{\boldsymbol{\varphi}^{\prime}(u)-\overline{\boldsymbol{\psi}}(u) \boldsymbol{\psi}^{\prime}(u)}{1+e^{\boldsymbol{\varphi}(u)}}\right] .
$$

The equivalence of (3.23) and (3.24) is intuitively clear, but we do not know a simple formal proof. It can be established order by order by expansing the exponential and evaluating the residues for each term.

After inserting the r.h.s. of (3.24) in (3.21) and integrating by parts we see that, in accord with the original Dorey-Tateo prescription, the expression for the excited partition function is obtained by extending the integration, originally along the real axis, by a contour encircling the roots $\mathbf{w}^{-\gamma}$,

$$
\mathcal{Z}(L, R, \mathbf{w})=\left\langle\exp \int_{\mathbb{R} \cup \mathbf{w}^{-\gamma}} \frac{d u}{2 \pi}\left(\partial_{u} \overline{\boldsymbol{\varphi}} \log \left(1+e^{-\boldsymbol{\varphi}}\right)-\frac{\overline{\boldsymbol{\psi}} \boldsymbol{\psi}^{\prime}}{1+e^{\boldsymbol{\varphi}}}\right)\right\rangle_{L, R} .
$$

It happens that in the case of periodic boundary conditions the naïve prescription (3.21)-(3.22) gives the correct result. This is an accident. In more general situations, as in the case of open boundaries in the mirror channel, the operators on the r.h.s. of (3.23) or (3.24) contribute non-trivially to the subleading order, as it was recently discovered in $[17,30]$. In the periodic case the subleading contributions cancel completely.

\section{Path integral and localisation}

The most transparent formulation of the effective QFT is given by a path integral. As we will see later, the integration measure does not need any regularisation.

To formulate the path integral for the effective QFT one should add, in addition to the fields $\varphi, \bar{\varphi}, \psi, \bar{\psi}$, two extra pairs of fields, $\rho, \bar{\rho}$ and $\vartheta, \bar{\vartheta}$, which define respectively the bosonic and fermionic propagators. The expectation value (3.17) is then given by

$$
\begin{aligned}
Z(L, R)= & \int \mathcal{D}[\text { fields }] e^{-\mathcal{A}[\text { fields }]}, \\
-\mathcal{A}[\text { fields }]= & \int_{-\infty}^{\infty} \frac{d u}{2 \pi}\left(\log \left(1+e^{-\varphi}\right) \partial_{u} \bar{\varphi}-\frac{\bar{\psi} \partial_{u} \psi}{1+e^{\varphi}}+\left(\bar{\varphi}-\bar{\varphi}^{\circ}\right) \rho+\bar{\rho}\left(\varphi-\varphi^{\circ}\right)+\bar{\vartheta} \psi+\bar{\psi} \vartheta\right) \\
& -\iint_{-\infty}^{\infty} \frac{d u}{2 \pi} \frac{d v}{2 \pi} \tilde{\phi}(u, v)(\rho(u) \bar{\rho}(v)+\bar{\vartheta}(v) \vartheta(u)),
\end{aligned}
$$

where $\tilde{\phi}(u, v)$ denotes the scattering phase in the mirror channel,

$$
\tilde{\phi}(u, v)=\frac{1}{i} \log \tilde{S}(u, v)
$$


As the action of the path integral (4.1) is linear in the fields $\bar{\varphi}$ and $\bar{\rho}$, we write it in the form

$$
\mathcal{A}=-\int \frac{d u}{2 \pi} \bar{\varphi}^{\circ} \rho+\int \frac{d u}{2 \pi}\left(\bar{\varphi} \mathcal{A}_{\bar{\varphi}}+\bar{\rho} \mathcal{A}_{\bar{\rho}}\right)+\text { fermions. }
$$

The fields $\bar{\varphi}$ and $\bar{\rho}$ play the role of Lagrange multipliers and can be integrated out, giving functional delta-functions. The path integral thus localises to the critical point $\mathcal{A}_{\bar{\rho}}=\mathcal{A}_{\bar{\varphi}}=0$,

$$
\begin{array}{ll}
\mathcal{A}_{\bar{\rho}}=0 \Rightarrow & \varphi(u)=\varphi^{\circ}(u)+\int \frac{d v}{2 \pi} \rho(v) \tilde{\phi}(v, u), \\
\mathcal{A}_{\bar{\varphi}}=0 \Rightarrow & \rho(u)=\partial_{u} \log \left(1+e^{-\varphi(u)}\right) .
\end{array}
$$

After excluding $\rho$ and integrating by parts, one obtains the TBA equation for the pseudoenergy $\varepsilon(u) \equiv \varphi(u)$,

$$
\varphi(u)=\varphi^{\circ}(u)-\int \frac{d v}{2 \pi} \log \left(1+e^{-\varphi(v)}\right) \tilde{K}(v, u) .
$$

Furthermore the gaussian fluctuations of the bosons and the fermions completely cancel and the partition function is given by the exponent of the critical action

$$
\mathcal{F}(L, R)=-\mathcal{A}_{\text {crit }}=L \int_{-\infty}^{\infty} \frac{d u}{2 \pi} \tilde{p}^{\prime}(u) \log \left(1+e^{-\varphi(u)}\right) .
$$

This paraphrases Pozsgay's argument [16] about the absence of an $O(1)$ term in the free energy.

The localisation implies a stronger statement, namely that there are no $1 / L$ corrections to the free energy, see e.g. [31]. The localisation of the path integral is generically a consequence of a fermionic symmetry of the measure. In our case the fermionic symmetry is generated by the nilpotent operators

$$
\begin{aligned}
& Q(u)=\bar{\psi} \frac{\delta}{\delta \varphi}+\bar{\varphi} \frac{\delta}{\delta \psi}+\bar{\rho} \frac{\delta}{\delta \vartheta}+\bar{\vartheta} \frac{\delta}{\delta \rho} \\
& \bar{Q}(u)=\psi \frac{\delta}{\delta \bar{\varphi}}+\varphi \frac{\delta}{\delta \bar{\psi}}+\rho \frac{\delta}{\delta \bar{\vartheta}}+\theta \frac{\delta}{\delta \bar{\rho}}
\end{aligned}
$$

satisfying the algebra $Q^{2}=\bar{Q}^{2}=0, Q \bar{Q}=\bar{Q} Q=2$. The action in (4.1) takes the form of a sum of a term which does not produce quantum effects and a $Q$-exact "localisation term",

$$
\mathcal{A}=\mathcal{A}^{\circ}+\int \frac{d u}{2 \pi} \mathrm{Q}(u) \mathcal{B}, \quad \mathcal{A}^{\circ}=-\int \frac{d u}{2 \pi} \bar{\varphi}^{\circ} \rho, \quad \mathcal{B}=\bar{Q} \mathcal{A} .
$$

The odd functional $\mathcal{B}$ is given by the integral

$$
\mathcal{B}=-\int \frac{d v}{2 \pi}\left(\psi^{\prime} \log \left(1+e^{-\varphi}\right)+\psi \rho+\vartheta\left(\varphi-\varphi^{\circ}\right)\right)+\int \frac{d u}{2 \pi} \frac{d v}{2 \pi} \rho(v) \tilde{\phi}(v, u) \vartheta(u) .
$$

The path integral with such an action localises at its critical point. The standard localisation argument ${ }^{5}$ is that the second term can be multiplied by any constant $t$ without changing the path integral:

$$
\mathcal{A} \rightarrow \mathcal{A}^{\circ}+t \mathrm{QB}
$$

\footnotetext{
${ }^{5}$ See e.g. the recent review [32].
} 
The modified path integral

$$
\mathcal{Z}_{t}=\int e^{-\mathcal{A}^{\circ}-t \mathrm{Q} \mathcal{B}}
$$

does not depend on the coupling $t$,

$$
\frac{\partial \mathcal{Z}_{t}}{\partial t}=\int e^{-\mathcal{A}^{\circ}-t \mathrm{QB}}(-\mathrm{Q} \mathcal{B})=\int \mathrm{Q}\left(e^{-\mathcal{A}^{\circ}-t \mathrm{QB}} \mathcal{B}\right)=0
$$

where it is used the fact that the measure is $Q$-invariant to perform the integration by parts. Taking the limit $t \rightarrow \infty$ one obtains

$$
\mathcal{Z}=\left.e^{-\mathcal{A}^{\circ}}\right|_{\mathrm{QB}=0}
$$

The condition $\mathrm{QB}=0$ selects the critical point (4.4).

The above argument holds also in the case of an excited state in the physical channel, in which case the action contains an extra term $i \bar{\varphi}\left(w_{1}^{-\gamma}\right)+\ldots+i \bar{\varphi}\left(w_{N}^{-\gamma}\right)$ and critical point determined by the TBA equation in presence of an excited state,

$$
\varphi(u)=\varphi^{\circ}(u)-i \sum_{k=1}^{N} \tilde{\phi}\left(u, w_{k}^{-\gamma}\right)-\int_{-\infty}^{\infty} \frac{d v}{2 \pi} \log \left(1+e^{-\varphi(v)}\right) \tilde{K}(v, u) .
$$

Here we used that, thanks to the localisation property, the functional variables can be considered as holomorphic functions of the variable $u$ and thus can be analytically continued in a strip around the real axis. The critical action is given by the well known expression for the excited state free energy

$$
\mathcal{F}(L, R, \mathbf{w})=-L \sum_{j=1}^{N} E\left(w_{j}\right)+\int \frac{d u}{2 \pi} \log \left(1+e^{-\varphi(u)}\right) \partial_{u} \bar{\varphi}^{\circ}(u) .
$$

In the case of open boundary conditions one obtains a one-loop exact path integral, with the gaussian fluctuations contributing to the subleading term in the free energy. The one-loop contribution then is given by the sum over the trees with a cycle in the graph expansion developed in ref. [33].

\section{Example: the Sinh-Gordon model}

For concrete integrable models of QFT, the path integral representation (4.1) can be transformed further to reveal more of the integrable structure. Here we examine the Sinh-Gordon model, described by the Euclidean action

$$
\mathcal{A}=\int d^{2} x\left[\frac{1}{4 \pi}(\nabla \phi)^{2}+\frac{2 \mu^{2}}{\sin \pi b^{2}} \cosh (b \phi)\right] .
$$

The model depends on the dimensonless parameter $b$ and the coupling constant $\mu$ of dimension $[\mathrm{mass}]^{1+b^{2}}$. This is the simplest non-compact integrable model. The thermodynamic Bethe Ansatz for the sinh-Gordon model was formulated by Al. Zamolodchikov [34] and S. Lukyanov [35], who derived functional equations for the TBA pseudo-energy and related 
functions. More rigorous approach based on an integrable lattice regularization was developped in $[27,36]$. Some recent developments concern the computation of the one-point functions [37] and diagonal form factors [38].

As a scattering theory, the sinh-Gordon model involves a single massive relativistic particle whose mass $m$ is determined by the coupling constant $\mu$. If the energy and the momentum are parametrised by the rapidity $u$ as

$$
p(u)=m \sinh \pi u, \quad E(u)=m \cosh \pi u,
$$

then the two-particles S-matrix is given by a product of two "universal" S-matrices,

$$
S(u)=S_{0}(u+i a / 2) S_{0}(u-i a / 2), \quad S_{0}(u)=\frac{e^{\pi u}-i}{e^{\pi u}+i},
$$

with the shift parameter $a$ related to the dimensionless parameter $b$ by

$$
a \equiv\left(1-b^{2}\right) /\left(1+b^{2}\right)
$$

The "universal" scattering kernel

$$
K_{0}(u, v) \equiv-i \partial_{u} \log S_{0}(u)=\frac{\pi}{\cosh \pi(u-v)}
$$

satisfies the difference equation

$$
K_{0}(u+i / 2)+K_{0}(u-i / 2)=2 \pi \delta(u) .
$$

It proves to be very helpful to represent the shifts of the rapidities as the result of the action of powers of the analytic difference operator

$$
\mathbb{D}=\exp \left(\frac{i}{2} \frac{\partial}{\partial u}\right)
$$

Then the relations (5.3) and (5.6) can be written as

$$
S(u)=e^{\left(\mathbb{D}^{a}+\mathbb{D}^{-a}\right) \log S_{0}(u)}=S_{0}(u)^{\mathbb{D}^{a}+\mathbb{D}^{-a}}, \quad\left(\mathbb{D}+\mathbb{D}^{-1}\right) K_{0}(u)=2 \pi \delta(u) .
$$

Formally the sinh-Gordon scattering kernel $K=-i \partial_{u} \log S=\left(\mathbb{D}^{a}+\mathbb{D}^{-a}\right) K_{0}$ represents the operator

$$
\mathbb{K}=\frac{\mathbb{D}^{a}+\mathbb{D}^{-a}}{\mathbb{D}+\mathbb{D}^{-1}}
$$

acting on the meromorphic functions defined in the rapidity complex plane. We can also invert this operator paying attention to the fact that both $\mathbb{K}$ and $\mathbb{K}^{-1}$ have non-trivial null spaces. For example, the energy and the momentum (5.2) belong to the null space of the operator $\mathbb{D}+\mathbb{D}^{-1}$.

Now let us formulate the path integral of the effective QFT for the sinh-Gordon model. Replacing in eq. (4.1) the expression for the sinh-Gordon scattering matrix in the form (5.8), 
we have

$$
\begin{aligned}
\mathcal{Z}^{\mathrm{ShG}}(L, R)= & \int[\text { fields }] e^{-\mathcal{A}[\text { fields }]}, \\
\mathcal{A}[\text { fields }]= & \int_{-\infty}^{\infty} \frac{d u}{2 \pi}\left(-\log \left(1+e^{-\varphi}\right) \bar{\varphi}^{\prime}-\frac{\bar{\psi} \psi^{\prime}}{1+e^{\varphi}}+\left(\bar{\varphi}-\bar{\varphi}^{\circ}\right) \rho+\left(\varphi-\varphi^{\circ}\right) \bar{\rho}+\bar{\vartheta} \psi+\bar{\psi} \vartheta\right) \\
& -i \int \frac{d u}{2 \pi} \frac{d v}{2 \pi}(\bar{\rho}(u) \rho(v)-\vartheta(v) \bar{\vartheta}(u))\left(\mathbb{D}^{a}+\mathbb{D}^{-a}\right) \log S_{0}(u-v)
\end{aligned}
$$

with

$$
\varphi^{\circ}(u)=R m \cosh \pi u, \quad \bar{\varphi}^{\circ}(u)=L m \sinh \pi u .
$$

The components of $\rho$ and $\bar{\rho}$ in the null space of the operator $\mathbb{D}+\mathbb{D}^{-1}$ impose the asymptotic conditions at infinity

$$
\varphi(u) \simeq \varphi^{\circ}(u), \quad \bar{\varphi}(u) \simeq \bar{\varphi}^{\circ}(u) \quad(u \rightarrow \infty) .
$$

We will impose instead these boundary conditions by hand and simultaneously restrict the functional integration in $\rho$ and $\bar{\rho}$ to the space orthogonal to the null space of the operator $\mathbb{D}+\mathbb{D}^{-1}$. We assume that the fluctuating field variables can be analytically continued in the strip $|\Im u|<1 / 2$, so that they can be acted on by the shift operators $\mathbb{D}^{ \pm 1}$.

The property (5.8) of the universal kernel can be used to simplify the integral. After a change of variables

$$
\bar{\rho}=\left(\mathbb{D}+\mathbb{D}^{-1}\right) \chi, \quad \bar{\vartheta}=\left(\mathbb{D}+\mathbb{D}^{-1}\right) \xi,
$$

the action takes a quasi-local form

$$
\begin{aligned}
\mathcal{A}[\text { fields }]= & \int \frac{d u}{2 \pi}\left(-\log \left(1+e^{-\varphi}\right) \bar{\varphi}^{\prime}-\frac{\bar{\psi} \psi^{\prime}}{1+e^{\varphi}}+\left(\varphi-\varphi^{\circ}\right)\left(\mathbb{D}+\mathbb{D}^{-1}\right) \chi\right) \\
& +\int \frac{d u}{2 \pi}\left(\left(\bar{\varphi}-\bar{\varphi}^{\circ}\right) \rho+\bar{\psi} \vartheta+\psi\left(\mathbb{D}+\mathbb{D}^{-1}\right) \xi\right) \\
& -\int \frac{d u}{2 \pi} \frac{d v}{2 \pi}\left(\rho\left(\mathbb{D}^{a}+\mathbb{D}^{-a}\right) \chi+\vartheta\left(\mathbb{D}^{a}+\mathbb{D}^{-a}\right) \xi\right) .
\end{aligned}
$$

The fields $\rho$ and $\vartheta$ can be integrated out giving the constraints $\bar{\varphi}=\bar{\varphi}^{\circ}+\left(\mathbb{D}^{a}+\mathbb{D}^{-a}\right) \chi$ and $\bar{\psi}=\left(\mathbb{D}^{a}+\mathbb{D}^{-a}\right) \xi$. As a result the action for the path integral simplifies to

$$
\begin{aligned}
\mathcal{A}[\varphi, \chi, \psi, \xi]= & -\int \frac{d u}{2 \pi} \partial_{u} \bar{\varphi}^{\circ} \log \left(1+e^{-\varphi}\right) \\
& +\int \frac{d u}{2 \pi}\left[\varphi\left(\mathbb{D}+\mathbb{D}^{-1}\right) \chi-\log \left(1+e^{-\varphi}\right)\left(\mathbb{D}^{a}+\mathbb{D}^{-a}\right) \chi\right] \\
& +\int \frac{d u}{2 \pi}\left[\psi\left(\mathbb{D}+\mathbb{D}^{-1}\right) \xi-\frac{1}{1+e^{\varphi}} \psi\left(\mathbb{D}^{a}+\mathbb{D}^{-a}\right) \xi\right]
\end{aligned}
$$

with asymptotic condition $\varphi \simeq \varphi^{\circ}$ at $u \rightarrow \infty$. The action is linear in $\chi$ and the path integral localises to the critical point which is the solution of the discrete Liouville equation

$$
\left[\mathbb{D}+\mathbb{D}^{-1}\right] \varphi=-\left(\mathbb{D}^{a}+\mathbb{D}^{-a}\right) \log \left(1+e^{-\varphi}\right) .
$$


This equation determines the pseudoenergy $\varphi$ uo to a periodic function in $u \rightarrow u+i$. The ambiguity is lifted by the condition that $\varphi \rightarrow \varphi^{\circ}$ at infinity. With this condition the saddle point equation gives TBA equation

$$
\varphi(u)=R E(u)-\int \frac{d v}{2 \pi} \log \left(1+e^{-\varphi(v)}\right) K(v, u) .
$$

Sometimes functional equations as (5.18) offer a more effective tool for accessing the solution than the integral TBA equation. The method of the "Quantum Spectral Curve" [39, 40], based on a set of functional equations for Baxter's $Q$-functions, proved to be very successful in the AdS/CFT integrability. The $Q$-function, introduced for the sinh-Gordon model in [34] and [35], is related to the critical value of the bosonic field by $\varphi=\left(\mathbb{D}^{a}+\mathbb{D}^{-a}\right) \log Q$. The discrete Liouville equation implies for the following functional identity for the $Q$-function,

$$
Q^{\mathbb{D}+\mathbb{D}^{-1}}=1+Q^{\mathbb{D}^{a}+\mathbb{D}^{-a}}
$$

which can be formulated as a bilinear equation

$$
Q(u+i / 2) Q(u-i / 2)-Q(u+i a / 2) Q(u-i a / 2)=1 .
$$

Al. Zamolodchikov [34] showed using these functional equations that $Q(u)$ and $Y(u)=$ $e^{\varphi(u)}$ are entire functions of $u$ and that the $Q$-system (5.21) implies periodicity for the T-functions defined as

$$
T(u)=Q^{-1}\left(\mathbb{D}^{1-a}+\mathbb{D}^{a-1}\right) Q
$$

namely $\left(\mathbb{D}^{a}-\mathbb{D}^{-1}\right) T=0$, which implies that $T(u)$ is a single-valued function of $\zeta^{2}=$ $e^{2 \pi\left(1+b^{2}\right) u}$ with essential singularities at $\zeta=0$ and $\zeta=\infty$.

\section{Concluding remarks}

In this paper we gave the Thermodynamical Bethe Ansatz a more familiar to particle physicists formulation based on an effective QFT defined in the rapidity plane (or Riemann surface). The elementary excitations of the effective QFT correspond to particles wrapping the two main cycles of the torus. The connected vacuum Feynman diagrams for the effective QFT are in one-to-one correspondence with the graphs in the exact cluster expansion for the free energy worked out in $[11,12]$.

An intriguing feature of the exact operator representation of the sum over mirror wrapping particles, eqs. (3.13)-(3.14), is that the two logarithmic factors in the integrand have similar form, although they were derived by completely different arguments. Integrating by parts and shifting the integration variable as $u \rightarrow u^{-\gamma}$ gives the original integrand with $\mathbf{A}$ and $\mathbf{B}$ exchanged. This remarkable fact means that if we take the opposite limit, $R$ large and $L$ finite, and write a similar free-field representation, it will have the same form as (3.13) up to the choice of the integration contour, which this time should encircle the singularities of the factor $\log (1+\mathbf{B})$. It is not excluded that the representation (3.13) can be used as a starting point to attack the problem with finite $R$. 
We restricted ourselves to the simplest possible scattering theory with only one neutral particle. This restriction is not very important and there are no conceptual difficulties in generalising the construction to more interesting cases of non-diagonal scattering and bound states in the physical/mirror channels, once the Bethe-Yang equations are diagonalised by the Nested Bethe Ansatz [41]. There is however a subtle point which is not yet well understood. The diagonalisation involves auxiliary particles of magnonic type with zero momentum and energy. The auxiliary magnons should be treated in exactly the same way as the momentum-carrying particles, although this is justified only if the number of the momentum-carrying particles is large. Nevertheless this prescription seems to reproduce correctly the Lüscher corrections for periodic [42] and even for open [33] boundary conditions. Apart of this subtlety the generalisation is straightforward. A pair of wrapping operators will be associated with each node of the Dynkin graph. The partition function (possibly with excited states in the physical channel) can be reformulated in terms of a path integral, which localises to a critical point determined by the TBA equations.

The effective QFT description might be useful in computing a class of correlation functions in the AdS/CFT integrability. For example, the "simplest" four-point function studied in [43-47] can be formulated as an effective CFT of real fermions living on the rapidity Riemann surface $[48,49]$. In this simplest case the world sheet splits into two or more disks (octagons).

In general, the world sheet for a correlation function of $n$ single-trace operators has the topology of a sphere with $n$ punctures. Such world sheet hosts a negative curvature $(2-n) \pi$ distributed among $n-2$ curvature defects represented by the so called hexagon operators [50]. In this case the $\mathbf{A}$ and the $\mathbf{B}$ operators of the effective QFT will be associated with a system of $A$-cycles and $B$-cycles of the world sheet, the $A$-cycles connecting pairs of punctures and the $B$-cycles connecting pairs of hexagons.

In [50] and in a series of subsequent works the correlation function is expressed as an integral over the mirror particles wrapping the $B$-cycles. The integral is divergent as a consequence of the infinite length of the $A$-cycles and requires regularisation. For a single pair of mirror particles, this divergency has been taken under control by a point-splitting regularisation of the integral over the rapidities [51], but it is not clear how to proceed in the case of several mirror particles. Assuming that the effective CFT description can be generalised to this case, the on-shell condition associated with each (asymptotically large) $A$-cycle would give an unambiguous prescription about how to regularise the contribution of arbitrary number of mirror magnons.

\section{Acknowledgments}

The author thanks Emil Nissimov and Svetlana Pacheva for useful comments and Zoltan Bajnok, Shota Komatsu, Didina Serban and Valentina Petkova for important critical remarks on the manuscript. 


\section{A Conventions about the scattering in physical and mirror kinematics}

We start with the infinite-volume description where the dispersion relation between the momentum and the energy of the particles in is parametrised by an intrinsically complexvalued rapidity variable $u$,

$$
p=p(u), E=E(u),
$$

with $p(-u)=-p(u)$ and $E(-u)=E(u)$. The mirror transformation exchanging the space and the time is obtained as an analytic continuation in the rapidity along some path $\gamma$. We adopt the notations of [51] and denote by $u^{\gamma}$ the endpoint of the path which can live in a different sheet of the rapidity Riemann surface. For a pedagogical explanation see [52]. For a relativistic field theory one can set

$$
p=m \sinh (\pi u), \quad E=m \cosh (\pi u), \quad u^{n \gamma}=u+i n / 2 \quad \text { (relativistic QFT). }
$$

The momentum and the energy of the mirror theory are given by

$$
\tilde{p}(u)=-i E\left(u^{\gamma}\right), \quad \tilde{E}(u)=-i p\left(u^{\gamma}\right)
$$

and should take real values for $u$ real. The square of the mirror transformation $\gamma$ is the crossing transformation $\gamma^{2}$ exchanging particles and anti-particles. The two-particle scattering matrix $S(u, v)$ is a unimodular function, analytic in the physical strip and satisfying the conditions

$$
\begin{aligned}
S(u, v) S(v, u) & =1 & & \text { (unitarity), } \\
S\left(u, v^{-\gamma}\right) S\left(u, v^{\gamma}\right) & =1 & & \text { (crossing unitarity). }
\end{aligned}
$$

We do not suppose that $S(u, v)$ is a function of the difference of the rapidities. We also assume that

$$
S(u, u)=-1,
$$

since this is the case with all known scattering theories excepr the free bosons. The S-matrix in the mirror channel

$$
\tilde{S}(u, v)=S\left(u^{\gamma}, v^{\gamma}\right)
$$

shares the same properties.

\section{B Operator representation of the Gaudin determinant}

Here we will give the operator representation of the determinant of any matrix of the form

$$
G_{i k}=G_{i} \delta_{i k}-K_{k i}^{-}, \quad G_{i} \equiv D_{i}+\sum_{k=1}^{m} K_{i k}^{+} .
$$

The determinant of the matrix (B.1) can be represented as an expectation value of a product of $m$ operators composed of the bosonic and fermionic gaussian variables, which 
we denote respectively by $\bar{\varphi}_{j}, \varphi_{j}$ and $\bar{\psi}_{j}, \psi_{j}$, with $j=1, \ldots, m$. If the non-zero bosonic and fermionic correlations are given by

$$
\left\langle\bar{\varphi}_{j}\right\rangle=D_{j}, \quad\left\langle\bar{\varphi}_{j} \varphi_{k}\right\rangle=K_{j k}^{+}, \quad\left\langle\psi_{j} \bar{\psi}_{k}\right\rangle=K_{j k}^{-},
$$

then $^{6}$

$$
\operatorname{det} G=\left\langle\prod_{j=1}^{m}\left(\bar{\varphi}_{j}-\psi_{j} \bar{\psi}_{j}\right) e^{\varphi_{j}}\right\rangle .
$$

The fermionic and the bosonic variables will be considered as specialisations of the bosonic and fermionic fields whose correlation functions given by the scattering phases

$$
\langle\overline{\boldsymbol{\varphi}}(u) \varphi(v)\rangle=-\tilde{\phi}(u, v), \quad\langle\overline{\boldsymbol{\psi}}(u) \boldsymbol{\psi}(v)\rangle=\tilde{\phi}(u, v) .
$$

Namely

$$
\varphi_{j}=-\varphi\left(u_{j}\right)+\varphi^{\circ}\left(u_{j}\right), \bar{\varphi}_{j}=\bar{\varphi}^{\prime}\left(u_{j}\right), \psi_{j}=\boldsymbol{\psi}\left(u_{j}\right), \bar{\psi}_{j}=\bar{\psi}^{\prime}\left(u_{j}\right),
$$

and for the correlators

$$
K_{j k}^{ \pm}=K^{ \pm}\left(u_{j}, u_{k}\right)
$$

where

$$
\begin{aligned}
\tilde{K}^{+}(u, v) & \equiv-\left\langle\bar{\varphi}^{\prime}(u) \varphi(v)\right\rangle=\partial_{u} \tilde{\phi}(u, v), \\
\tilde{K}^{-}(u, v) & \equiv\left\langle\boldsymbol{\psi}^{\prime}(u) \bar{\psi}(v)\right\rangle=-\partial_{u} \tilde{\phi}(v, u)
\end{aligned} .
$$

Then (B.3) yields

$$
\operatorname{det} G\left(\mathbf{u}^{\mathbf{r}}\right) \prod_{k=1}^{m} e^{-r_{k} \varphi^{\circ}\left(u_{k}\right)}=\left\langle\prod_{k=1}^{m}\left[\overline{\boldsymbol{\varphi}}^{\prime}\left(u_{j}\right)-r_{j} \overline{\boldsymbol{\psi}}\left(u_{j}\right) \boldsymbol{\psi}^{\prime}\left(u_{j}\right)\right] e^{-r_{k} \boldsymbol{\varphi}\left(u_{k}\right)}\right\rangle .
$$

This is the general formula in which one should specify the form of the scattering phases. For periodic boundary conditions

$$
\begin{aligned}
\tilde{\phi}(u, v) & =\frac{1}{i} \log \tilde{S}(u, v), & \tilde{K}^{+}(u, v) & =\tilde{K}^{-}(u, v)=\tilde{K}(u, v), \\
\left\langle\bar{\varphi}^{\prime}(u) \varphi(v)\right\rangle & =-\tilde{K}(u, v), & \left\langle\boldsymbol{\psi}^{\prime}(u) \overline{\boldsymbol{\psi}}(v)\right\rangle & =\tilde{K}(u, v) .
\end{aligned}
$$

Open Access. This article is distributed under the terms of the Creative Commons Attribution License (CC-BY 4.0), which permits any use, distribution and reproduction in any medium, provided the original author(s) and source are credited.

\section{References}

[1] A.B. Zamolodchikov, Thermodynamic Bethe Ansatz in relativistic models. Scaling three state Potts and Lee-Yang models, Nucl. Phys. B 342 (1990) 695 [InSPIRE].

[2] A.B. Zamolodchikov, From tricritical Ising to critical Ising by thermodynamic Bethe ansatz, Nucl. Phys. B 358 (1991) 524 [inSPIRE].

\footnotetext{
${ }^{6} \mathrm{~A}$ detailed derivation is presented in [12] where it was used to give a compact proof of the matrix-tree theorem.
} 
[3] A.B. Zamolodchikov, TBA equations for integrable perturbed $\mathrm{SU}(2)_{k} \times \mathrm{SU}(2)_{l} / \mathrm{SU}(2)_{k+l}$ coset models, Nucl. Phys. B 366 (1991) 122 [INSPIRE].

[4] C.-N. Yang and C.P. Yang, Thermodynamics of a one-dimensional system of bosons with repulsive delta-function interaction, J. Math. Phys. 10 (1969) 1115 [INSPIRE].

[5] N. Beisert et al., Review of AdS/CFT Integrability: An Overview, Lett. Math. Phys. 99 (2012) 3 [arXiv: 1012.3982] [INSPIRE].

[6] M. Lüscher, Volume Dependence of the Energy Spectrum in Massive Quantum Field Theories. 1. Stable Particle States, Commun. Math. Phys. 104 (1986) 177 [InSPIRE].

[7] G. Kato and M. Wadati, Bethe Ansatz Cluster Expansion Method for Quantum Integrable Particle Systems, J. Phys. Soc. Jap. 73 (2004) 1171.

[8] G. Kato and M. Wadati, Direct calculation of thermodynamic quantities for the Heisenberg model, J. Math. Phys. 43 (2002) 5060 [cond-mat/0212325].

[9] G. Kato and M. Wadati, Graphical representation of the partition function of a one-dimensi onal $\delta$-function Bose gas, J. Math. Phys. 42 (2001) 4883 [cond-mat/0212323].

[10] G. Kato and M. Wadati, Partition function for a one-dimensional $\delta$-function Bose gas, Phys. Rev. E 63 (2001) 036106 [cond-mat/0212321].

[11] I. Kostov, D. Serban and D.-L. Vu, TBA and tree expansion, Springer Proc. Math. Stat. 255 (2017) 77 [arXiv: 1805.02591] [INSPIRE].

[12] I. Kostov, D. Serban and D.-L. Vu, Boundary TBA, trees and loops, Nucl. Phys. B 949 (2019) 114817 [arXiv: 1809.05705] [INSPIRE].

[13] D.-L. Vu and T. Yoshimura, Equations of state in generalized hydrodynamics, SciPost Phys. 6 (2019) 023 [arXiv: 1809.03197] [INSPIRE].

[14] D.-L. Vu, Cumulants of conserved charges in GGE and cumulants of total transport in GHD: exact summation of matrix elements?, arXiv:1909.08852 [INSPIRE].

[15] F. Woynarovich, O(1) contribution of saddle point fluctuations to the free energy of Bethe Ansatz systems, Nucl. Phys. B 700 (2004) 331 [cond-mat/0402129] [INSPIRE].

[16] B. Pozsgay, On O(1) contributions to the free energy in Bethe Ansatz systems: The Exact g-function, JHEP 08 (2010) 090 [arXiv: 1003.5542] [INSPIRE].

[17] Y. Jiang, S. Komatsu and E. Vescovi, Structure Constants in $\mathcal{N}=4$ SYM at Finite Coupling as Worldsheet g-Function, arXiv:1906.07733 [INSPIRE].

[18] N.A. Nekrasov and S.L. Shatashvili, Quantization of Integrable Systems and Four Dimensional Gauge Theories, in Proceedings, 16th International Congress on Mathematical Physics (ICMP09): Prague, Czech Republic, August 3-8, 2009, pp. 265-289, 2009, DOI [arXiv: 0908.4052] [INSPIRE].

[19] J. Balog, Field theoretical derivation of the TBA integral equation, Nucl. Phys. B 419 (1994) 480 [INSPIRE].

[20] A.M. Polyakov and P.B. Wiegmann, Theory of nonabelian Goldstone bosons in two dimensions, Phys. Lett. 131B (1983) 121 [INSPIRE].

[21] E. Ogievetsky, N. Reshetikhin and P. Wiegmann, The principal chiral field in two-dimension and classical Lie algebra, NORDITA-84/38. 
[22] L.D. Faddeev and N.Yu. Reshetikhin, Integrability of the Principal Chiral Field Model in (1+1)-dimension, Annals Phys. 167 (1986) 227 [INSPIRE].

[23] C. Destri and H.J. De Vega, Unified approach to thermodynamic Bethe Ansatz and finite size corrections for lattice models and field theories, Nucl. Phys. B 438 (1995) 413 [hep-th/9407117] [INSPIRE].

[24] C. Destri and H.J. de Vega, Non-linear integral equation and excited-states scaling functions in the sine-Gordon model, Nucl. Phys. B 504 (1997) 621 [hep-th/9701107] [INSPIRE].

[25] P. Zinn-Justin, Quelques applications de l'ansatz de Bethe, Ph.D. Thesis, Paris University, France (1998).

[26] D. Volin, Quantum integrability and functional equations: Applications to the spectral problem of AdS/CFT and two-dimensional $\sigma$-models, J. Phys. A 44 (2011) 124003 [arXiv: 1003.4725] [INSPIRE].

[27] J. Teschner, On the spectrum of the Sinh-Gordon model in finite volume, Nucl. Phys. B 799 (2008) 403 [hep-th/0702214] [INSPIRE].

[28] S.L. Lukyanov, Free field representation for massive integrable models, Commun. Math. Phys. 167 (1995) 183 [hep-th/9307196] [INSPIRE].

[29] F. Woynarovich, On the normalization of the partition function of Bethe Ansatz systems, Nucl. Phys. B 852 (2011) 269 [arXiv:1007.1148] [InSPIRE].

[30] Y. Jiang, S. Komatsu and E. Vescovi, Exact Three-Point Functions of Determinant Operators in Planar N =4 Supersymmetric Yang-Mills Theory, Phys. Rev. Lett. 123 (2019) 191601 [arXiv: 1907.11242] [INSPIRE].

[31] T.R. Klassen and E. Melzer, Purely Elastic Scattering Theories and their Ultraviolet Limits, Nucl. Phys. B 338 (1990) 485 [inSPIRE].

[32] V. Pestun, Review of localization in geometry, J. Phys. A 50 (2017) 443002 [arXiv: 1608.02954] [INSPIRE].

[33] D.-L. Vu, I. Kostov and D. Serban, Boundary entropy of integrable perturbed $\mathrm{SU}(2)_{k} W Z N W$, JHEP 08 (2019) 154 [arXiv: 1906.01909] [INSPIRE].

[34] A.B. Zamolodchikov, On the thermodynamic Bethe ansatz equation in sinh-Gordon model, J. Phys. A 39 (2006) 12863 [hep-th/0005181] [INSPIRE].

[35] S.L. Lukyanov, Finite temperature expectation values of local fields in the sinh-Gordon model, Nucl. Phys. B 612 (2001) 391 [hep-th/0005027] [INSPIRE].

[36] A.G. Bytsko and J. Teschner, Quantization of models with non-compact quantum group symmetry: Modular XXZ magnet and lattice sinh-Gordon model, J. Phys. A 39 (2006) 12927 [hep-th/0602093] [INSPIRE].

[37] S. Negro and F. Smirnov, On one-point functions for sinh-Gordon model at finite temperature, Nucl. Phys. B 875 (2013) 166 [arXiv:1306.1476] [INSPIRE].

[38] Z. Bajnok and F. Smirnov, Diagonal finite volume matrix elements in the sinh-Gordon model, Nucl. Phys. B 945 (2019) 114664 [arXiv:1903.06990] [inSPIRE].

[39] N. Gromov, V. Kazakov, S. Leurent and D. Volin, Quantum Spectral Curve for Planar $\mathcal{N}=4$ Super-Yang-Mills Theory, Phys. Rev. Lett. 112 (2014) 011602 [arXiv:1305.1939] [InSPIRE].

[40] N. Gromov, V. Kazakov, S. Leurent and D. Volin, Quantum spectral curve for arbitrary state/operator in $A d S_{5} / C F T_{4}, J H E P 09$ (2015) 187 [arXiv:1405.4857] [INSPIRE]. 
[41] P.P. Kulish and N.Yu. Reshetikhin, Diagonalization of GL(N) invariant transfer matrices and quantum $N$-wave system (Lee model), J. Phys. A 16 (1983) L591 [InSPIRE].

[42] C. Ahn, Z. Bajnok, D. Bombardelli and R.I. Nepomechie, TBA, NLO Lüscher correction and double wrapping in twisted AdS/CFT, JHEP 12 (2011) 059 [arXiv:1108.4914] [INSPIRE].

[43] F. Coronado, Bootstrapping the simplest correlator in planar $\mathcal{N}=4$ SYM at all loops, arXiv: 1811.03282 [INSPIRE].

[44] F. Coronado, Perturbative four-point functions in planar $\mathcal{N}=4 S Y M$ from hexagonalization, JHEP 01 (2019) 056 [arXiv: 1811.00467] [INSPIRE].

[45] A.V. Belitsky and G.P. Korchemsky, Exact null octagon, arXiv:1907.13131 [INSPIRE].

[46] T. Bargheer, F. Coronado and P. Vieira, Octagons I: Combinatorics and Non-Planar Resummations, JHEP 08 (2019) 162 [arXiv: 1904.00965] [INSPIRE].

[47] T. Bargheer, F. Coronado and P. Vieira, Octagons II: Strong Coupling, arXiv: 1909.04077 [INSPIRE].

[48] I. Kostov, V.B. Petkova and D. Serban, The Octagon as a Determinant, JHEP 11 (2019) 178 [arXiv: 1905.11467] [INSPIRE].

[49] I. Kostov, V.B. Petkova and D. Serban, Determinant formula for the octagon form factor in $\mathcal{N}=4$ SYM, Phys. Rev. Lett. 122 (2019) 231601 [arXiv:1903.05038] [InSPIRE].

[50] B. Basso, S. Komatsu and P. Vieira, Structure Constants and Integrable Bootstrap in Planar $N=4 S Y M$ Theory, arXiv:1505.06745 [INSPIRE].

[51] B. Basso, V. Goncalves and S. Komatsu, Structure constants at wrapping order, JHEP 05 (2017) 124 [arXiv: 1702.02154] [INSPIRE].

[52] S. Komatsu, Lectures on Three-point Functions in $N=4$ Supersymmetric Yang-Mills Theory, in Proceedings, Les Houches Summer School: Integrability: From Statistical Systems to Gauge Theory: Les Houches, France, vol. 106, 2019, DOI [arXiv:1710.03853] [INSPIRE]. 